\title{
Odontogenic Factors Associated with Food Impaction in Patients Attending Periodontics OPD of Universal College of Medical Sciences
}

\author{
Adhikari $\mathrm{K}^{1}$, Dutta $\mathrm{K}^{2}$, Bali $\mathrm{H}^{3}$ \\ ${ }^{1}$ Assistant Professor, Department of Periodontology and Oral Implantology, College of Dental Surgery-BPKIHS, \\ Dharan, Nepal \\ ${ }^{2}$ Lecturer, Department of Orthodontics and Dentofacial Orthopedics, College of Dental Surgery-UCMS, Bhairahawa, \\ Nepal \\ ${ }^{3}$ Lecturer, Department of Oral Medicine and Radiology, Kathmandu University School of Medical Sciences, Dhulikhel, \\ Nepal
}

\begin{abstract}
Introduction: Food impaction is a common problem experienced by almost everyone during their lifetime. It is believed that the interproximal wedging of food normally occurs due to loss of integrity of the proximal contact and its location along with the contour of the marginal ridges and lack of papilla fill. The objective of the study was to assess the role of odontogenic factors for food impaction and identify the odontogenic factors most prevalent in patients attending patients attending Periodontics OPD of Universal College of Medical Sciences.

Materials and Methods: A total of hundred subjects with natural dentition from second molar to second molar in both the maxillary and mandibular arches were enrolled in this cross-sectional study. Clinical parameters like pocket depth, bleeding index and plaque index was recorded. The integrity of contact was recorded using a dental floss. A study cast was fabricated for each subject. Presence of plunger cusps along with marginal ridge relationship, position of tooth in the arch and contiguity of contact points were analyzed on it.

Results: A significant association of plaque index score and food impaction was found $(p=0.03)$. Of all the factors, loose contact showed a slightly significant positive correlation with plaque index score $(\mathrm{r}=0.19, \mathrm{p}=0.05)$.

Conclusions: Amongst all the odontogenic factors, loose contact is positively correlated with plaque index and food impaction is significantly associated with plaque score. The odds of food impaction were significant with the brushing technique employed and frequency of tooth brushing in the subjects.
\end{abstract}

Key words: Dental care; Food impaction; Open contact.

\section{Introduction}

$\mathrm{F}$ ood impaction is defined as the forceful wedging of food into the interproximal space by chewing pressure or the forcing of food inter-proximally by tongue or cheek pressure. ${ }^{1}$

Conflict of Interest: No

\section{*Corresponding Author}

Khusbu Adhikari, Assistant Professor

Department of Periodontology and Oral Implantology, College of Dental Surgery-BPKIHS, Dharan, Nepal

E-mail:adhkhush@gmail.com
It is believed that the interproximal wedging of food normally occurs due to loss of integrity and location of the proximal contact and the contour of the marginal ridges along with lack of papilla fill. ${ }^{2-4}$ It can affect the periodontium in many ways and lead to pain, halitosis, inflammation, hypersensitivity, cervical caries, recession and may infect the pulp and even cause tooth loss. ${ }^{3,5,6}$ It is a common cause for periodontal diseases. ${ }^{7}$

There are limited studies on a different population in the past to explore this relationship. ${ }^{2,3}$ Hence, this warrants further investigation of those factors in our part of the world. The aim of this 
study is to assess the role of odontogenic factors for food impaction in our population along with identifying the factors most prevalent for food impaction in the same.

\section{Materials and Methods}

This cross-sectional study was conducted in the Department of Periodontics and Oral Implantology, Universal College of Medical Sciences (UCMS), Bhairahawa, Nepal from August 2018 to December 2018. Approval from the Institutional Review Committee was obtained (UCMS/IRC/ 74/18). The sample size was calculated using the formula:

$\mathrm{n}=\mathrm{N}^{*} \mathrm{z}_{\alpha}^{2} \mathrm{p}^{*}(1-\mathrm{p})$

$(\mathrm{N}-1) \mathrm{e}^{2}+\mathrm{z}_{\alpha}^{2} * \mathrm{p}(1-\mathrm{p})$

A total of hundred subjects with natural dentition from second molar to second molar in both the maxillary and mandibular arches were enrolled in this study. Subjects with acute oral diseases, any known systemic diseases, undergone orthodontic treatment for two years or with a temporary provisional restoration were excluded from the study.

Demographic data including oral hygiene practice were recorded for all subjects giving a written informed consent. A complete periodontal examination was carried out for each subject using a mouth mirror, University of North Carolina (UNC) -15 periodontal probe and dental floss. Plaque Index ${ }^{8}$, Papillary Bleeding Index ${ }^{9}$, Pocket depth (millimetres) ${ }^{10}$ were clinical parameters recorded for individual subjects.

Following which, each proximal area was assessed and the findings recorded for the presence or absence of interproximal calculus tactilely, proximal restorations with or without overhangs was noted if proximal restoration was present. Food impaction was scored as present or absent and was determined by the presence of fibrous food wedged interproximally. ${ }^{7}$ Finally, integrity of the contact was checked twice with a double strand by a dental floss. ${ }^{11}$ Each contact was recorded as tight; when a definite resistance to the passage of floss was felt. As loose contact; when minimal resistance was felt and absence of resistance was recorded as open contact.

An impression of both the maxillary and mandibular arches was made with alginate for each subject and a study cast with a base was fabricated. Marginal ridge relationship, position of tooth in the arch and contiguity of contact points was evaluated on study cast using the ABO (American Board of Orthodontics) objective system for grading dental casts. ${ }^{12}$

Data were entered into an Excel Sheet and analysed using SPSS V22.0, IBM, Chicago, IL, USA. Demographic variables are presented as frequency. Chi-Square test was carried out for determining the association of factors responsible for the food impaction. Pearson Correlation test was applied to assess the relationship between the odontogenic factors and food impaction. A $p-$ value of $\leq 0.05$ was considered statistically significant.

\section{Results}

Eighty-six subjects out of hundred were aged between 21 to 25 years. There were 12 subjects in the age range of 26-30 years, while one subject each from the age group of 31-35 years and 36-40 years respectively. Out of hundred subjects, 69 were female and 31 were male. Maximum subjects $(n=84)$ had a mixed dietary habit whereas 16 of them were vegetarian.

Food impaction was present in 42 and absent 58 subjects. Oral hygiene practice of all the subjects was recorded. Among the types of toothbrush, most of the subjects were using medium toothbrush $(n=49)$, soft $(n=46)$, ultrasoft $(n=4)$ and hard $(n=1)$. The frequency of brushing once a day was recorded for 47 
subjects and twice a day for remaining 53 subjects. Modified Bass technique $(n=33)$ was the most commonly used technique of brushing followed by horizontal( $n=29), \quad \operatorname{scrub}(n=21)$, $\operatorname{Bass}(\mathrm{n}=8), \operatorname{vertical}(\mathrm{n}=7)$ and $\operatorname{circular}(\mathrm{n}=2)$. Sixteen subjects changed their toothbrush within 1 to 2 months, 67 between 3 to 4 months and 17 between 5 to 6 months. Oral hygiene aid was used only by 12 subjects and not used by the rest $(\mathrm{n}=88)$.

Table 1 describes the occurrence of various factors on the cast in subjects with presence and absence of food impaction. Table 2 shows a significant association of plaque index score and food impaction with a $p$ value of 0.03 . When we further applied Pearson's correlation between plaque index score and food impaction, we found positive correlation between them where, $\mathrm{r}=0.18, \mathrm{p}=0.06$.

Pocket depth is seen slightly increased in presence of food impaction $2.77 \pm 0.22$ as compared with absence of food impaction $2.72 \pm 0.29$ but the difference is not significant (Table 3). No significant difference was noted for papillary bleeding index in both the groups (Table 3).

When different clinical parameters were correlated with different integrity of contact, no correlation was found with presence or absence of food impaction. However, plaque index score showed a slightly significant positive correlation with loose contact where, $r=0.19$, $\mathrm{p}=0.05$ (Table 4).

In Table 5, we calculated odds ratio for different parameters of oral hygiene practice for food impaction. The odds of food impaction were significant with the brushing technique employed $(\mathrm{OR}=0.74, \mathrm{p}=0.02)$ and frequency of tooth brushing $(\mathrm{OR}=0.58, \mathrm{p}=0.18)$. Whereas, use of oral hygiene aid and change of toothbrush or type of toothbrush and did not show significant values.

Table 1: Frequency of odontogenic factors and their odds ratio on cast between presence and absence of food impaction.

\begin{tabular}{|l|c|c|c|c|}
\hline \multirow{2}{*}{$\begin{array}{l}\text { Cast Parameters } \\
\text { (n) }\end{array}$} & \multicolumn{2}{|c|}{ Food Impaction } & \multirow{2}{*}{ Odds ratio } & p value \\
\cline { 2 - 3 } & Absent & Present & & \\
\hline Presence of Open Contacts & 55 & 51 & 1.03 & 0.79 \\
\hline Change in Cervicoincisal Inclination & 14 & 6 & 0.77 & 0.37 \\
\hline Change in Buccolingual Inclination & 226 & 142 & 0.95 & 0.65 \\
\hline Occlusal/ Incisal Facets & 15 & 16 & 0.96 & 0.40 \\
\hline Loss of marginal Ridge Integrity & 7 & 1 & 0.29 & 0.26 \\
\hline
\end{tabular}

Table 2: Association of Food impaction with Calculus and Plaque index scores.

\begin{tabular}{|l|l|c|c|c|}
\hline \multirow{2}{*}{ Parameters } & \multicolumn{3}{|c|}{ Food Impaction } & \multirow{2}{*}{ p value } \\
\cline { 2 - 4 } & & Absent & Present & \multirow{2}{*}{0.23} \\
\hline Calculus & Absent & 8 & 9 & \\
& Present & 50 & 33 & 0.03 \\
\hline \multirow{2}{*}{ Plaque Index } & Excellent & 2 & 8 & \\
\cline { 2 - 4 } & Good & 27 & 30 & \\
\cline { 2 - 4 } & Fair & 6 & 4 & \\
\hline
\end{tabular}


Table 3: Value of Pocket depth and Papillary Bleeding Index in presence and absence of food impaction.

\begin{tabular}{|c|c|c|c|}
\hline \multirow{2}{*}{ Parameters } & \multicolumn{2}{|c|}{ Food Impaction } & \multirow{2}{*}{ p value } \\
\cline { 2 - 3 } & $\begin{array}{c}\text { Absent } \\
\text { (Mean } \pm \text { SD) }\end{array}$ & $\begin{array}{c}\text { Present } \\
\text { (Mean } \pm \text { SD) }\end{array}$ & \\
\hline Pocket Depth & $2.72 \pm 0.29$ & $2.77 \pm 0.22$ & 0.45 \\
\hline Papillary Bleeding Index & $0.17 \pm 0.04$ & $0.17 \pm 0.03$ & 0.92 \\
\hline
\end{tabular}

Table 4: Pearson's Correlation of different clinical parameters with different integrity of contact.

\begin{tabular}{|c|c|c|c|c|c|c|}
\hline \multirow{3}{*}{ Parameters } & \multicolumn{6}{|c|}{ Integrity of Contact } \\
\hline & \multicolumn{2}{|c|}{ Tight } & \multicolumn{2}{|c|}{ Loose } & \multicolumn{2}{|c|}{ Open } \\
\hline & $\mathbf{r}$ & p & $\mathbf{r}$ & p & $\mathbf{r}$ & p \\
\hline Pocket Depth & -0.06 & 0.54 & 0.12 & 0.20 & -0.001 & 0.99 \\
\hline Papillary Bleeding Index & 0.03 & 0.75 & 0.08 & 0.42 & -0.08 & 0.38 \\
\hline Calculus & 0.08 & 0.42 & -0.13 & 0.17 & -0.01 & 0.92 \\
\hline Food Impaction & -0.15 & 0.12 & 0.15 & 0.13 & 0.08 & 0.39 \\
\hline Plaque Index & 0.03 & 0.74 & 0.19 & 0.05 & -0.16 & 0.10 \\
\hline
\end{tabular}

Table 5: Odds ratio of Oral hygiene practice with presence and absence of food impaction.

\begin{tabular}{|l|c|c|}
\hline Variables of Oral Hygiene practice & Odds Ratio & p value \\
\hline Toothbrush type & 1.09 & 0.80 \\
\hline Brushing technique & 0.74 & 0.02 \\
\hline Frequency of Brushing & 0.58 & 0.18 \\
\hline Change of toothbrush & 0.83 & 0.61 \\
\hline Use of Oral Hygiene Aid & 2.12 & 0.22 \\
\hline
\end{tabular}

\section{Discussion}

Food impaction into interproximal spaces can traumatize the gingival tissues, creating an area favourable to bacterial growth and multiplication. Subsequently, a release of toxic products may result in soft tissue and bone destruction. ${ }^{13}$ Food impaction was reported in 42 of the total subjects included in this study.

Proximal contact loss seems to induce food impaction along with other occlusal morphology factors, such as uneven marginal ridges and prominent opposing cusps. ${ }^{14}$ The occurrence of various odontogenic factors recorded on the cast showed no significant value for food impaction in our subjects (Table 1). Similarly, when different integrity of contact was correlated with clinical parameters, only plaque index score showed a significantly slight positive correlation with loose contact (Table
2). A number of studies reported a positive association between open contacts and presence of periodontal disease. ${ }^{4,15}$ Whereas another study reported that the significance of open contacts on periodontal disease is very minimal. ${ }^{16}$ Despite of conflicting reports linking open contacts with periodontal disease, Consensus report by 1999 International World Workshop for classification of periodontal disease and conditions has classified open contacts under anatomic tooth factors that predispose/modify periodontal disease. ${ }^{17}$

Hancock ${ }^{3}$ et al. determined the relationship of interdental contacts on periodontal status of naval recruits. Results revealed no significant relationship between contact type and gingival index or probing depth but a significant relationship was seen between food impaction and contact type and between food impaction 
and probing depth. These findings add on to the concept that food impaction contributes to periodontal disease. While the exact role of a deficient interproximal contact may not be clear, open contacts leading to food impaction are often uncomfortable to the patient, and tight interproximal contacts are generally considered important for gingival health.

In the present study, Papillary bleeding index was almost similar in the presence or absence of food impaction but a slight difference in pocket depth in presence and absence of food impaction was noted, which was not significant. Prichard ${ }^{13}$ found that in 82 patients with 106 interproximal intrabony pockets, all showed some factor associated with food impaction in the area of the lesion. Although, lose or open proximal contacts clinically seem to be contributing factors to periodontal pocket formation but literature proposes different view on the subject. ${ }^{3}$ This may be attributed to oral hygiene levels of the different study populations.

A significant association was seen between food impaction and plaque index in the subjects (Table 2). The position or inclination of teeth can be factors that predispose the periodontium to plaque accumulation and subsequent loss of attachment. A change in buccolingual inclination of teeth in the arch was noted for maximum subjects in this study. While studies show that areas of periodontium adjacent to maligned teeth can be maintained in a state of health, in situations where meticulous oral hygiene is not practiced, periodontal disease can occur. When adequate oral hygiene was maintained, no difference was demonstrated in periodontal breakdown at sites with deficient proximal contacts compared to satisfactory sites. ${ }^{2}$

With regard to oral hygiene practices, 53 of the total subjects brushed twice daily and 33 of them employed modified bass method of tooth brushing. There were a larger number of subjects who changed their toothbrush within 3 to 4 months. This indicates that subjects have adequate knowledge of oral hygiene practices. The odds of food impaction are significant based on the oral hygiene practice, especially the technique and frequency of brushing (Table 5). Suomi et al. ${ }^{18}$ reported that persons having frequent prophylaxes and personal instructions in oral hygiene had lower plaque and calculus scores, less gingival inflammation, and a slower rate of apical migration of the epithelial attachment than persons not receiving these benefits.

\section{Conclusions}

One hundred subjects were examined clinically as well as their diagnostic cast was analysed to determine whether there is any relationship between various odontogenic factors and food impaction. The observations made were that Plaque index score and food impaction showed a positive correlation and slightly significant positive correlation with loose contact. The odds of food impaction were significant with the brushing technique employed and frequency of tooth brushing. Further longitudinal studies with a larger sample size will definitely add on value to our findings and help us in diagnosing and treating this most encountered problem in our practice.

\section{Acknowledgements}

We would like to express our sincere gratitude to Professor Dr. Anand Kumar, Principal, Universal College of Medical Sciences(UCMS) and Mr. Raju Dubey. We would like to acknowledge the immense help from students, interns and staff of Department of Periodontics and Oral Impalntology, UCMS, Bhairahawa for their support throughout the study.

\section{Financial Disclosure}

This study was supported by research grant from Universal College of Medical Sciences, Bhairahawa, Nepal. 


\section{References}

1. American Academy of Periodontology. Glossary of Periodontal Terms. $4^{\text {th }}$ Edition

2. Kepic TJ, O’Leary TJ. Role of marginal ridge relationships as an etiologic factor in periodontal disease. J Periodontol. 1978; 49: 570-5. https:// doi.org/10.1902/jop.1978.49.11.570

3. Hancock EB, Mayo CV, Schwab RR, Wirthlin MR. Influence of interdental contacts on periodontal status. J Periodontol. 1980; 51: 4459. https://doi.org/10.1902/jop.1980.51.8.445

4. Jernberg GR, Bakdash MB, Keenan KM. Relationship between proximal tooth open contacts and periodontal disease. J Periodontol. 1983; 54: 529-33. https://doi.org/10.1902/ jop.1983.54.9.529

5. van den Broek AM, Feenstra L, de Baat C. A review of the current literature on aetiology and measurement methods of halitosis. J Dent. 2007;35: 627-35. https://doi.org/10.1016/j. jdent.2007.04.009

6. Bidra AS. Nonsurgical management of inflammatory periimplant disease caused by food impaction: A clinical report. J Prosthet Dent. 2014;111: 96-100. https://doi.org/10.1016/j. prosdent.2013.06.017

7. Hirschfeld I. Food impaction. The Journal of the American Dental Association (1922). 1930 Aug 1;17(8):1504-28. https://scholar. google.com/scholar_lookup?journal=J+Am + Dent + Assoc\&title $=$ Food + impaction\&auth or $=$ I + Hirschfeld \& volume $=17 \&$ publication year $=1930 \&$ pages $=1504-1528 \&$

8. Silness J, Löe H. Periodontal disease in pregnancy. II. Correlation between oral hygiene and periodontal condition. Acta Odontol Scand. 1964; 22:112-35. https://doi. org/10.3109/00016356408993968

9. Saxer U, Turconi B, Elsässer C. Patient motivation with the papillary bleeding index. J Prev Dent. 1977; 4: 20-2. PMID: 351174
10. Tibbetts, L. S.: Use of diagnostic probes for detection of periodontal disease. J Am Dent Assoc. 1969 78: 549. DOI: 10.14219/jada. archive. 1969.0127

11. O'Leary, T. J., Baudell, M. C, and Bloomer, R. S.: Interproximal contact and marginal ridge relationships in periodontally healthy young males classified as to orthodontic status. J Periodontol.1975; 46: 6. DOI: 10.1902/ jop.1975.46.1.6

12. Casko JS, Vaden JL, Kokich VG, Damone J, James RD, Cangialosi TJ, et al. Objective grading system for dental casts and panoramic radiographs. American Board of Orthodontics. Am J Orthod Dentofacial Orthop. 1998;114:58999. DOI: 10.1016/s0889-5406(98)70179-9

13. Prichard, J.: A Technique for Treating Intrabony Pockets Based on Alveolar Process Morphology, Dental Clinics of North America, Philadelphia, W. B. Saunders Co.,March 1960.

14. Newell DH, John V, Kim SJ. A technique of occlusal adjustment for food impaction in the presence of tight proximal contacts. Oper Dent. 2002; 27:95-100.

15. Koral SM, Howell T.H, Jeffcoat MK. Alveolar bone loss due to open interproximal contacts in periodontal disease. J Periodontol.1981; 52(8): 447-50. 10.1902/jop.1981.52.8.447

16. Geiger A M, Wasserman B H, Turgeon L R. Relationship of occlusion and periodontal disease. Part VIII-Relationship of crowding and spacing to periodontal destruction and gingival inflammation. J Periodontol. 1974; 45(1):43-9. 10.1902/jop.1974.45.1.43

17. Blieden T M. Tooth related issues. Ann periodontal. 1999;4(1): 91-7. https://doi. org/10.1902/annals.1999.4.1.91

18. Suomi, J. D., Greene, H. C, Vermillion, J. R., Chang, J. J., and Leatherwood, E. C: The effect of controlled oral hygiene procedures on the progression of periodontal disease in adults: Results after two years. J Periodontol.1969;40: 416. https://doi.org/10.1902/jop.1969.40.7.416 